\title{
A Technological Infrastructure Design for a Pediatric Oncology Network
}

\author{
Aniello Murano \\ Università degli Studi di Napoli "Federico II" \\ Via Cinthia -80136 Napoli, Italy \\ murano@na.infn.it
}

\begin{abstract}
In Italy, the service of Pediatric oncology is very centralized and granted by high-specialized medical structures.

In the Italian Regione Campania there are just few of such medical centers that, in case of room availability, take care of the child from the diagnosis until his/her complete healing, providing all necessary treatments along with a number of assistance services both for the patient and his parents. Regione Campania centers are often congested, causing a migration of more than $35 \%$ of the young patients towards extraterritorial centers. Also, these medical centers are often far away from the place where the patients leave, constraining them to have recurrently long traveling, sometimes just for a routine test. However, in the Regione Campania there are a lot of facilities and hospitals with high technology equipments and qualified staff that, by means of an appropriate network organization based on a computer network infrastructure, could help during the children's protocol treatment and check and thus significantly contribute to improve the local and national pediatric oncology service.

This paper reports an evaluation research for the development, through an engineering approach based on a "hub and spoke" model, a complete and efficient information system that allows the organization and sharing, at different levels, of clinical information among high-specialized medical centers and the other entities.
\end{abstract}

\section{Categories and Subject Descriptors \\ J.3 Medical information systems.}

\section{General Terms}

Management, Performance, Design, Reliability, Experimentation, Human Factors.

\section{Keywords}

Medical, e-health, information system, pediatric oncology, hub and spoke, technological innovation, modern hospital.

\section{INTRODUCTION}

Oncology, and in particular pediatric oncology, is one of the most challenging areas involving the National Health System (NHS). While from one hand there is a modest but steady upward trend in the incidence of pediatric cancer, on the other hand there is a continuous coming out of upheaval scientific and technological innovations in the related areas of the diagnosis and treatment. Pediatric cancer healing rates strongly depend on the organization of the whole specialized healthcare system of reference. Usually, such a system is schematically based on four pillars: (a) family physicians; (b) diagnostic centers; (c) rehabilitation centers, longstay hospitals, socio-medical assistance centers; (d) high-tech and high assistance specialized hospitals. In order to avoid that specialized hospitals are inundated by improper requests and overwhelmed by providing low complexity health care services, which could be better provided in other structures of assistance with a lower citizens' discomfort and cost, in recent years it has been growing up everywhere the need of reorganizing the pediatric oncology service in a hierarchical and distributed model $[1,2,3]$. In this perspective, an organization model particularly efficient could be the "hub \& spoke" model [4,5]. In such a model, a specialized hospital (hub) is a component of a complex system of assistance and integrated in a network of services, in which the hub is a primary node, leaving to secondary centers (spokes) specific duties such as scheduled diagnosis, prevention, control and follow-up. A network of services organized in accordance with this model enjoys dynamism. In fact, the network architecture can be always improved on the basis of the development of new technologies, knowledge, skills, and management. The fair distribution of children among structures and operators providing an adequate (and economically balanced) volume of activities associated with a constant updating of knowledge and technologies ensures good quality of the NHS and a reduction of its expenses. In such a scenario, it is immediate to imagine the enormous contribution that may come from the use of a technologic infrastructure that takes care of the whole governance of the function of the whole network.

In Italy, some attempts of realizing a computer infrastructure for the pediatric oncology service have been already carried out, but no one of them is based on the "hub \& spoke" model. At an international level, an example of pediatric oncology network based on the "hub \& spoke" model is POGO (Pediatric Oncology Group of Ontario), although at the time it was designed it was thought for working without the support of the actual modern computer science technologies. In fact, Pogo was founded by a group of pediatric oncologists in 1983 [6].

In Campania, the Pediatric Oncology Service is provided by few high-specialized medical centers. These centers, in case of availability, take care of the child from the diagnosis until his/her complete healing, providing all necessary treatments along with a number of assistance services both for the patient and his parents. In order to improve patients' lifestyle, rationalize the use of regional health service resources and, thus, reduce the secondary costs of health assistance, it is commonly understood that there is a need for the creation in Campania (as well as elsewhere in Italy) of a shared pediatric oncology information system in which a number of currently completely independent entities (reference 
centers, diagnostic and/or assistance centers, patients, pediatricians, Cancer Registry, etc.) cooperate together. Such a system can be obtained by networking the various activities, through the implementation of integrated networks of services, in a way that, increasing levels of technical professional qualifications and technological equipments, even if physically placed in different territorial areas, are made available to all citizens independently from the access point to the system.

This paper reports an evaluative research for the development, through an engineering approach, of a complete and efficient information system, that allows the organization and sharing, at different levels, of clinical information among high-specialized medical centers and the other entities, by using innovative system and network technologies. The conceived information system is based on computer science technologies that integrates, harmonizes and rationalizes the different services of the NHS and used by children suffering of oncological diseases. Also, it complies with the guidelines given by the National and Regional Health Plan on the realization of integrated health information services. The new information system satisfies the clinical-scientific functionalities of the services it integrates, by using advanced technologies and new paradigms of analysis, design, and development of the applications, which allow, on one hand, the adoption of open, uniform, portable and interoperable systems and, on the other hand, fully comply with standardization and security criteria. A service provided by the NHS so integrated can support therapeutic continuity and proper treatments by allowing the recording of the disease clinical path, the possibility of updating it over time and planning its evolution and its sharing. Also, new technologies have been considered in order to provide automatic control of clinical data and management of emergency situations.

The new information system is conceived in such a way it can immediately and naturally transferable in the pediatric oncology health system of the Campania region. We believe that this can guarantee a significant improvement of the pediatric oncology service provided by the NHS. In particular it can allow:

- The implementation of a medical service distributed on the whole region, ensuring an improvement of the patients' lifestyle and a significant increase in the number of Campania's children which will be treated on the spot;

- Human resources rationalization. For example, by means of distance medical consultancy. As a side effect, in many cases, such rationalization will improve, at no cost, social assistance services provided by the NHS;

- Regional health centers rationalization, by preventing current congestion of high-specialization centers;

- The generation of coherent and reliable electronic data regarding all patients treated in regional health centers in support of monitoring and epidemiological centers of the NHS. For example, it is possible to localize areas with higher incidence of cancer diseases connected to particular events on the territory (as an example of application, one may think to the recent critical waste situation in the Regione Campania).

\section{THE SYSTEM ADD-ON}

The creation of a common and shared information system in Campania for the pediatric oncology service, based on advanced computer technologies, can surely allow a more efficient organization and sharing of clinical information, at different levels, between hospitals and the territory. In particular, such a system can support all the operators linked to the network, while performing their own tasks. This guarantees the continuity of the patient's therapy and the distribution of suited treatments by recording the clinical path along the time, with the possibility of updating it, planning its evolution, and sharing it among all centers interacting with the patient.

The realization of the networked information system adds several functionalities, and thus information services, to the NHS. In particular, the technology platform furnishes to its users the following services.

- to hub hospitals: to set up a shared Electronic Patient Record (EPR) for each patient, which allows to retrieve, in real-time, patient's coherent data, in an independent and concurrent way. Also, the EPR allows to recover aggregate data and statistics to analyze the course of treatments in med- and long-term. The web platform permits to coordinate with the spoke centers the most suited therapy, as well as to start the "first hosting center" procedure or alert a social service unit. Last but not least, the use of advanced computer science technologies (such as RfiD, Wifi, Palms) can support the medical team in their normal duties, to the aim of reducing the clinical treatment risk (for example, by providing support to prescriptions, alert functionalities, patient automatic identification, automatic matching between patient and chemotherapies, transfusion process control, etc).

- to spoke hospitals: to enter data of their pertinent patients and to update them every time scheduled therapies and diagnoses are carried out. Also, spoke hospital can have access to many of the functionalities described above for hub hospitals.

- to monitoring and epidemiologist centers of the NHS (for example to the Record Cancer Organizations): to enter coherent and reliable electronic data about all patients in treatment in the Campania region;

- to patients, their parents and pediatricians: to access to patients' data. In particular, they can see therapeutic protocols, preformed clinical trials, scheduled appointments, as well as to print documents and certificates. Also, the system provides automatic services such as such as receive a confirmation or cancellation email or SMS messages for clinical appointments.

- to external-region pediatric oncology computer network, and in particular to future national and international informatics hub \& spoke centers: to access aggregate data and share clinical treatment/expertise.

\section{DESIGN METHODS}

The design of the conceived computer network infrastructure has passed along with the following main steps:

- Network organization research and definition of system specifications. In this phase, an intensive study of the organization of the network service from a medical viewpoint has been carried out. During this phase, particular important have been the meetings carried out with the doctors and researchers working at the main hub hospital, in order to trace precisely all system requirements and services the system must guarantee.

- Preliminary considerations on legal aspects regarding sharing of healthcare information. To the aim of achieving interoperability among proprietary applications provided by different healthcare systems, a series of rules have been introduced by the 
competent organization. These rules have been deeply taken in consideration during the design of the information system.

- Preliminary considerations on required network capacity. In order to identify the more appropriate hardware platform for the implementation of the service, the amount of data that the network has to handle has been opportunely evaluated. In particular, the architecture of the network has been conceived in such a way it ensures an efficient, reliable, and secure flow of data.

- Security and privacy issues. Due to the high sensitivity of managed data, it has been important to ensure a high level of security, guaranteed by developing strict and secure access policies, data encryption, system protection by means of firewalls, and secure channels (HTTPS/SSL) for data transmission;

- Realization of the technological computer network through incremental releases. Most of the effort has been devoted on the evaluation on the more appropriate development (software and hardware) tools to be used as well as the most suitable system architecture to adopt. To reduce system design errors, automatic tools (test based and model checking based) [7] for error checking have been also considered.

Regarding the previously mentioned aspect of the regulation of sharing healthcare information, .it is commonly known that every computer application used by the Public Administration (PA) must obey a series of rules imposed by the competent authorities, in relation to the specific context in which the application operates. This applies in particular in the NHS where it is necessary to encourage "de facto" standards and those required by the law. These standards have been carefully taken into account while we have designed the network for this project. At international level, considerable resources are regularly engaged in standardization activities that are specific to the e-government of the NHS, by involving authorities, large public and private organizations, industries, and end-users (particularly the doctors and nurses). Currently, the two major organizations operating in the ICT, which are concerned in defining regulations for software (and telematics) in the NHS, are the CEN/TC251 and the HL7. Another important aspect of the ICT regulation in the health sector is a strong recommendation to the use of "open source" software, as it was done recently by the official "Three-year Plan 2008-2010 for the ICT in Public Administration" and by the European Commission under the plan of action e-Europe. This recommendation does not only have a technological value, but it also assumes political and economic meanings. In fact, the opportunity for the PA of using Free Software has positive effects on the local budget and, more in general, on the Italian deficit. The individuation of tools and technologies to be used in the implementation of the network system has been strongly guided by scalability and efficiency, in addition to the need of using open source products.

In technical details, all the above considerations have led us to implement the network system on a platform J2EE enterprise open source (JBoss on Tomcat), using standard Web Services for the distribution of services on the network. Also, we have chosen an implementation of the network architecture in a three tier model typical of modern medium and large web-based solutions (see Figure 1).

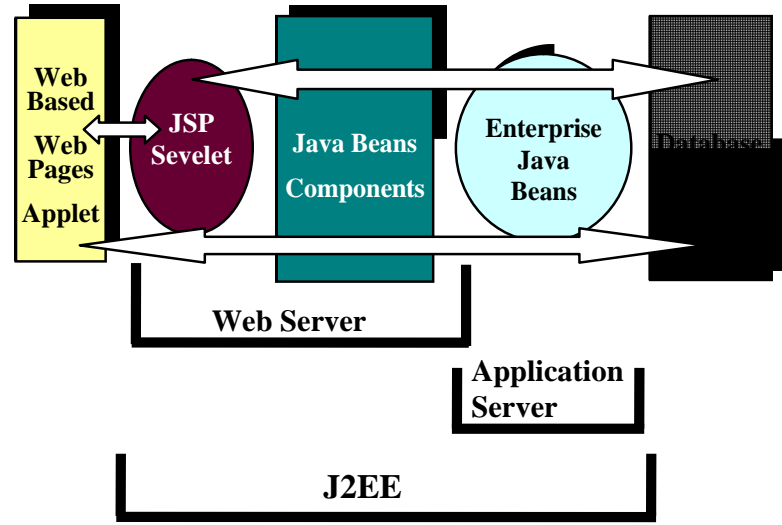

Figure 1. Web Tier e Application Tier

In this architecture, the system is typically divided on several machines based on the following breakdown levels:

- Presentation: it deals with the formatting of the page to be visualized through the browser. The interface is represented by a Web Server (HTTP);

- Application: it manages the application business logic. It corresponds to a series of integrated modules in an application server (which guarantees the execution of applications in a distributed context) for the generation of dynamic content (for example, J2EE modules on JBoss application server, connected to an engine JSP Tomcat).

- Data: it represents the level where data accessed by the business logic are stored. These data are stored on a database server.

The separation in several layers (physically in several machines) derives by the need of specializing servers, in accordance with the type of tasks they play, in order to optimize the system performance.

The machines used in presentation functionality (Web server) are used for non elaborative tasks and therefore they are not equipped with extremely capacity of calculation;

The machines used in application functionalities (application server) are used instead for elaborative tasks, whose difficulties strongly depend on the type of applications as well as they are implemented;

The machines used for data management functionalities (database) ensure a quickly answer to queries received from the application server.

The described task division thus allows us to handle different amount and type of work, executing an "ad-hoc" tuning depending on the type of task. This would clearly be not possible in the case in which all the features are collapsed on a single machine.

The choice of using more machines is also necessary for security reasons. Normally, web servers are arranged in an area easily accessible to users, while it is preferable to restrict the access to application server or to the database only to "experts". Such servers must therefore be allocated in appropriate network segments and the access should be protected by means of firewall. The database, which contains the patients' sensitive data, is configured in such a way that it can be accessed only trough an application server. For matters of reliability, the patients' data are stored on a server mounted in RAID modality. Moreover, to 
ensure greater efficiency of access to data, two DBMSs have been considered to work under the whole system. Figure 2 shows the whole technology infrastructure.

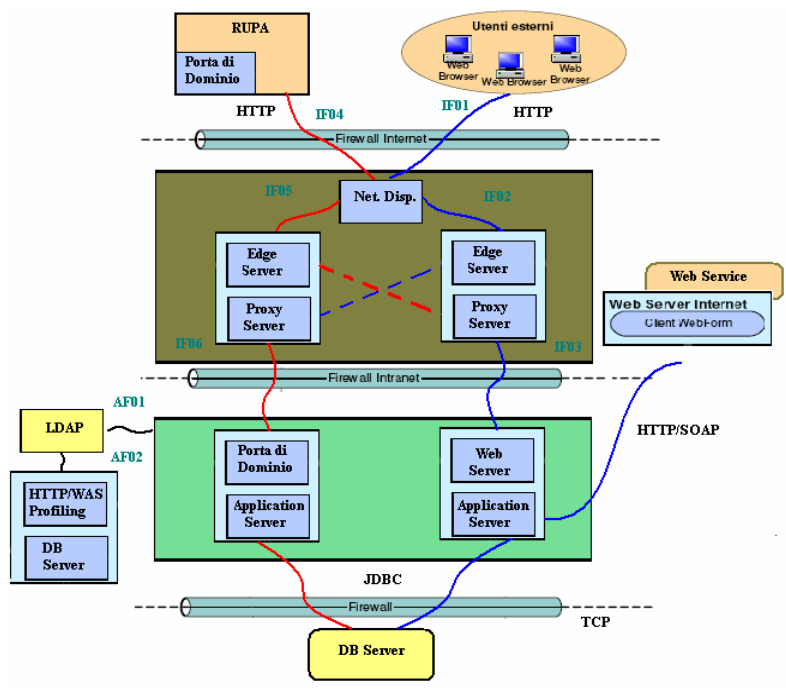

Figure 2: The Technologic infrastructure

\section{Conclusion}

We have reported in this paper a research devoted to the realization of a computer network infrastructure for the shared of the pediatric oncology service in the Italian Campania Region. This has been enforced by a research through an engineering approach, for a complete and efficient information system, that allows the organization and sharing, at different levels, of clinical information among high-specialized medical centers and the other entities, by using innovative computer science technologies. The conceived system provides two important issues: on one hand, it provides to the pediatric oncology community a technologically advances network infrastructure for the sharing of patients, information, and services. On the other hand, it allows the training of new researchers on the delicate aspect of merging computer science and medical abilities for the improvement of the National Health Service.

The system has been conceived by means of the following components:

- computer security component: it includes functionalities related to the authentication and to the management of the user profiles, as well as to the security during the transfer of information over the network. Also, this component allows common citizens to access public, non-sensitive information with no need for a special authorization;

- operational management component: it realizes the core management tasks for the information needed by the reference and satellite centers to provide their services;

- support and monitoring component: it provides functionalities to the reception centers and centers of service for the support of the cancer patient;

- clinical events tracing component: it holds the clinical history and all the significant clinical events involving the patient;
- informational component: it provides the consultant functionality of the information in the system through the Information Portal;

- local information system loading component: it enables the automatic transfer of data to the network system;

- monitoring and assessment component: it includes the necessary functionalities to provide the control bodies of the network with information and elements of assessment of the poles.

We believe that an important value of the conceived system resides also on the fact that the new pediatric oncology network service is immediately transferable into the clinical practice, as the service is easy to spread out within all institutions involved in the pediatric oncological service (in particular to pediatric hematological and oncology institutions) all over the Campania Region and beyond. In particular, the consideration that the project satisfies a very-hot need of both the operators of the National Health System and the users of the pediatric oncology service makes its transferability even more realistic.

Finally, the approach illustrated here could find applicability in the management of other diseases requiring collaborative care across institutions.

Aknowledgments. We wish to thank Prof. V. Poggi, the chief of the Pediatric Hematology-Oncology Unit of the AORN Santobono-Pausilipon of Naples, as well as Dr. G. Menna and Dr. $\mathrm{R}$. Parasole, for interesting discussions regarding the organization of the network service from a medical viewpoint. Being the most important clinical centre of the Campania region concerning pediatric oncology, the Department represents the clinical Hub Centre in the experimentation and in the implementation of the computer network healthcare system described in this paper. We also wish to thank Dr. A. Iorio for his technical help and support.

\section{REFERENCES}

[1] JF Bion and JE Heffner, Challenges in the care of the acutely ill. The Lancet. 2004 March 18; 363(9413): 970-977.

[2] JM Peppercorn, JC Weeks, EF Cook et al., Comparison of outcomes in cancer patients treated within and outside clinicaltrials: conceptual framework and structured review. Lancet. 2004 Jan 24; 363 (9405): 263-70.

[3] G Pearson, F Shann, P Barry et al., Should paediatric intensive care be centralised? Trent versus Victoria. The Lancet. 1997 Apr 26; 349(9060): 1213-1217.

[4] J. Grigsby and J.H. Sanders, Telemedicine: Where It Is and Where It's Going. Annals of Internal Medicine. 1998 July 15; 129, issue 2: $123-127$

[5] E Guzzanti, The hospital of the future: origins, evolution and perspectives. Recenti Prog Med, 2006 Nov;97(11):594-603.

[6] 'hittp:///www.pogo.ca/

[7] M. Clarke, O. Grumberg, and D.A. Peled, Model checking, MIT Press, Cambridge, MA, USA, 1999. 
This document was created with Win2PDF available at http://www.win2pdf.com.

The unregistered version of Win2PDF is for evaluation or non-commercial use only. This page will not be added after purchasing Win2PDF. 